Journal of

Dentistry and Oral Health

\title{
Surgical Guides for Dental Implants; a Suggested New Classification
}

\section{Salem $\mathrm{D}^{1, *}$, Mansour $\mathrm{MH}^{2}$}

${ }^{1}$ Dental/Oral and maxillofacial prosthetist. Private Practice Brisbane - Australia

${ }^{2}$ Oral and maxillofacial Surgeon Adjunct A. Prof Dental School University of Queensland

${ }^{*}$ Corresponding author: Salem D, Dental/Oral and maxillofacial prosthetist. Private Practice Brisbane - Australia, Email:mman0896@optusnet.com.au

Received Date: March 21, 2019 Accepted Date: April 28, 2019 Published Date: April 30, 2019

Citation: Salem D (2019) Surgical Guides for Dental Implants; a Suggested New Classification.J Dent Oral Health 6: 1-8.

\begin{abstract}
The concept of prosthetic-driven implantology has dominated the practice of implant placement in dentistry. The surgical guide/template has served as the communication tool for transfer of the treatment planning which is highly sophisticated at times. The literature is packed with articles of different designs and types of such guides without a clear, universally accepted classification. The treatment planning in implant dentistry involves a large team of different professionals who collaboratively execute the work. The lack of a classification of surgical guides makes the communication between this team rather difficult and cumbersome. A good classification of the surgical guides is therefore required. An extensive literature review via "Medline Ovid" from 1950 to 2019 about surgical guides in dental implants was performed. A new classification for the surgical guides incorporating all observations seen in published articles was devised. An instruction request card for a surgical guide from the requesting practitioner to the dental laboratory has, also, been designed. The use of this classification with the designed laboratory instruction guide has the potential to markedly enhance the communication and satisfaction between the various professionals dealing with dental implants.
\end{abstract}

Keywords: Surgical guide, classification, free guide, access guide, precision guide

\section{Introduction}

Surgical guides (stents) are templates that transfer information regarding tooth position(s) to the dentist prior to implant placement [1]. Surgical guides are manufactured in the dental laboratory, manually or using CAD/CAM technology mostly from acrylic resins, on instructions from the restorative dentist to assist with placement of the dental implant in its ideal restorative position. Holes are placed into the surgical guide to guide implant placement [2]. There appears to be a lack of a classification of different surgical guides and the literature is full of different types and ideas of such guides. Even though there has been an explosion in the use of computer-generated stereo-lithographic guides with high degrees of accuracy the need still exists for the use of less expensive conventional surgical guides. Not every practitioner has accessibility to the latest sophisticated technology. In many

C 2019 The Authors. Published by the JScholar under the terms of the Creative Commons Attribution License http://creativecommons.org/licenses/ by/3.0/, which permits unrestricted use, provided the original author and source are credited. parts of the world, dental implant rehabilitation commonly involves more than one practitioner and is more so true in complicated and difficult cases. The need still exists for adequate communication between the restoring practitioner, implant placing practitioner and the laboratory technician.

In the past, the implant site and direction were dictated by residual bone availability. The desire for a predictable fixed partial prosthesis led to the development of a newer concept of "prosthetically guided implantology" This concept establishes the correct implant position based on the planned definitive restoration and is achieved from the early planning phase [3].

Prior to that, in the early 90s, problems had been outlined in relation to maxillary implant placement that surgeons restored by eyeballing the dental implant placement, relative to the maxillary ridge. A line drawn from the anterior maxilla to the mandibular ridge became more acute as the mandible was opened. This change in angulation led the surgeon to lingually incline the dental implants. This inclination resulted in 
a prosthesis that had the anterior teeth cantilevered forward at a distance from the dental implant, making it very difficult to create an accurate framework and causing hygiene problems [4].

The philosophy behind successful aesthetic implant restoration became known as restoration-driven implant placement. This necessitated three parameters: first, developing an adequate volume of bone to support the implant as an extension of the restoration. Second, a sufficient volume of soft tissue to allow for adjustment of the prosthetic recipient site and third, a three-dimensional restoration-generated site allowing for a gingival margin of appropriate shape and tone $[5,6]$.

Ideal implant treatment planning requires close collaboration between the restorative dentist/prosthodontist and the oral surgeon/periodontist to determine the optimum placement of the implant in relation to the available bone and the prosthetic requirements. Surgical guides have evolved to be the mean that can aid in this treatment planning and the implementation of that plan [7]. Cases where the individual dentist performs the whole treatment would still benefit from a surgical guide as means of extra-oral planning of the case.

The ideal placement of dental implants should be determined by prosthetic parameters which depend on the position of a tooth in the arch. The exact positioning of the implant in bone with respect to location and angulation is often difficult to accurately match the prosthetic requirements [8].

Many different types of guides have been proposed, varying from the very simple designs, which may not provide the desired information to achieve pleasing results, to ones that are extremely complex which require a great deal of time and money to fabricate and are so precise as to not allow for any intraoperative changes that could arise due to local anatomy [7].

Surgical guides are used to improve the accuracy of implant placement and an ideal surgical guide should be stable, rigid, of limited size, easy to insert, transparent, modifiable and able to be sterilized [3].

Patients with residual dentition have more retention and better intraoral operative stability of the guide. In edentulous patients, the surgical reflection of the mucoperiosteal flap changes the fit of the template base on the underlying bone. Therefore, a maxillary template requires suitable support from the palate with extensions to the tuberosities. The mandible ideally requires a tripod configuration supported by two posterior extensions resting on both retromolar pads and by an anterior extension, a few millimeters wide, in the symphyseal area [9].

\section{Material of guide}

Traditionally, the more rigid surgical guides are fabricated with an auto-polymerizing acrylic resin or with composite resin. If a guide sleeve is used, it would be embedded in the acrylic resin. The guide will extend over the teeth for support and repositioning and this carries the risk of improper seating in a rigid guide if only a small area is not perfect. It has, therefore, been suggested to disclose all rigid guides with a thin layer of VPS (Vinyl Polysiloxane) impression material to adjust those poor contact areas. A less rigid, more flexible guide will usually fit completely even if minor discrepancies were present, however, most acrylic and composite resin guides will not gain retention from below the height of the covered teeth and will not be very stable during surgery. The use of rigid VPS as the main guide material has been advocated as a rapid, simple and relatively inexpensive alternative [10].

It can be technically difficult to position a surgical guide in the edentulous jaw or in patients with limited dentition. It has been suggested, in such cases, to use bone anchor pins or transitional implants to gain further stability of the surgical guide [9].

\section{Classification of Surgical Guides}

When discussing surgical stents or guides, there appears to be a reasonable variability in the literature regarding the nature and the types of stents used. There does not appear to be any strong consensus into classifying or defining different types of surgical guides.

In 1987, Balshi and Garver [11], attempted to describe different types of surgical guide stents. They mentioned that there were three basic surgical guide stents used in implant placement:

1. The fully edentulous.

2. The partially edentulous (removable partial denture design).

3. Partially edentulous tooth-supported design.

In this classification, they really resorted to the dentition status of the patient as the main parameter. They described the fully edentulous guide stent, as being also subdivided into two types - one, that provides a general guide to the area of dental implant placement and one that provides a specific guide to the location and angulation of each dental implant requiring placement. The general guide stent is almost a duplication of a transitional denture.

Stumpel [10], advocated a different classification. He utilized a conceptual approach to describing different types of surgical guides. This approach included three different concepts: 
The simplistic, non-limiting surgical guide, followed by the partially limiting surgical guide and finally, the completely limiting surgical guide.

The non-limiting surgical guide is one which will provide the operator an indication as to where the prosthesis needs to go in relation to the implant site. It will then be the decision of the operator to decide on the rest of the parameters regarding the angulation and accurate positioning of the implant.

The partially limiting design is one where there is some form of a guide sleeve that will direct only the first drill used for the preparation of the implant site, whereas the rest of the preparation will continue to be done freehand by the surgeon.

The completely limiting design is one that will restrict all the instruments used for drilling the implant site by that guide, including the buccolingual and mesiodistal planes, in addition to drill stops limiting the depth of the preparation and hence the final positioning of the prosthetic component.

\section{Methods}

An extensive literature search was performed on Medline from 1950 to 2019 to identify any proposed classifications for surgical guides used for dental implant placement. Different published designs of surgical guides were reviewed to try and form a consensus on a possible, comprehensive classification.

\section{Proposed Classification of Surgical Guides}

In essence, there are three types of surgical guides: a free guide, an access guide, and a precision guide. The name immediately implies the desired function of that guide. The "free guide" would be to show where the tooth center point

\section{DESIGN SUPPORT}

is. The "access guide" would guide the first drill only; whereas the precision guide would guide the whole drilling sequence. Once the restoring practitioner decides on the type of guide then the support during the surgical phase comes next. This will depend on the dentition and required stability and accuracy of the guide. There are 4 possibilities there, namely: tooth supported, tissue supported, both tooth and tissue supported, or tissue supported with an accessory fixation for edentulous cases. To cover for some of the designs that currently exist the access and precision guides can have fixed guidance or removable guidance where the guiding mechanism can be removed. With 3 designs and 4 supports there would be 12 potential guides. It is unlikely, though, to require a free or access guide with tissue support and accessory fixation. Accordingly, possible guides would be:

1) Free, tooth supported guide

2) Free, tooth and tissue supported guide

3) Free, tissue supported guide

4) Access, tooth supported guide

5) Access, tooth, and tissue supported guide

6) Access, tissue supported guide

7) Precision, tooth supported guide

8) Precision, tooth, and tissue supported guide

9) Precision, tissue supported guide

10)Precision, tissue supported with accessory fixation guide

Some clinical examples are presented to clarify the applicability of the proposed classification. Figure ' 1 ' demonstrates rehabilitation of an edentulous mandible with multiple implants where the position for an insertion was left to the time of surgery. The guide is designed to show where the first dill would go into the mandibular arch in the labial side of the guide.
- $\quad$ Free

- Access

-With fixed guidance

-With removable guidance

- Precision

-With fixed guidance

-With removable guidance
- $\quad$ Tooth supported only

- $\quad$ Tissue supported only

$\mathrm{X}$

- Tooth and tissue supported

- Tissue supported with added fixation 


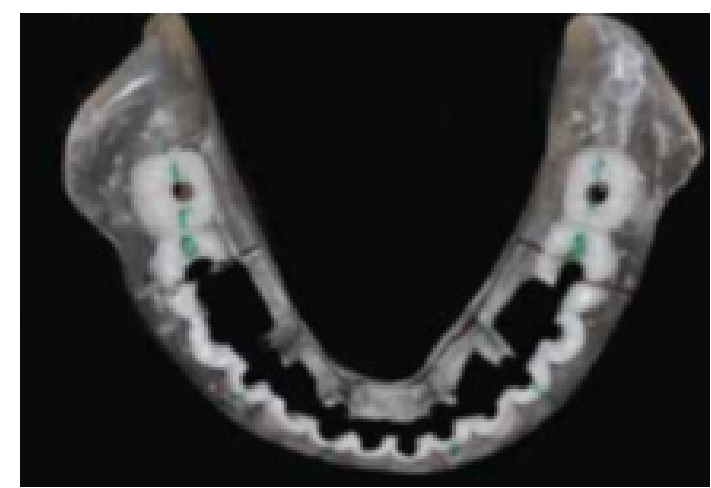

Figure 1. Lower mandibular Access, tissue supported guide

The part of the guide that covers the anterior and premolar dentition would be classified as an access guide and the support is from the soft tissues classifying it as an "access, tissue supported guide."

In the following case, missing tooth ' 37 'was rehabilitated with an implant. A surgical guide was prepared from acrylic as shown in figure 2 .

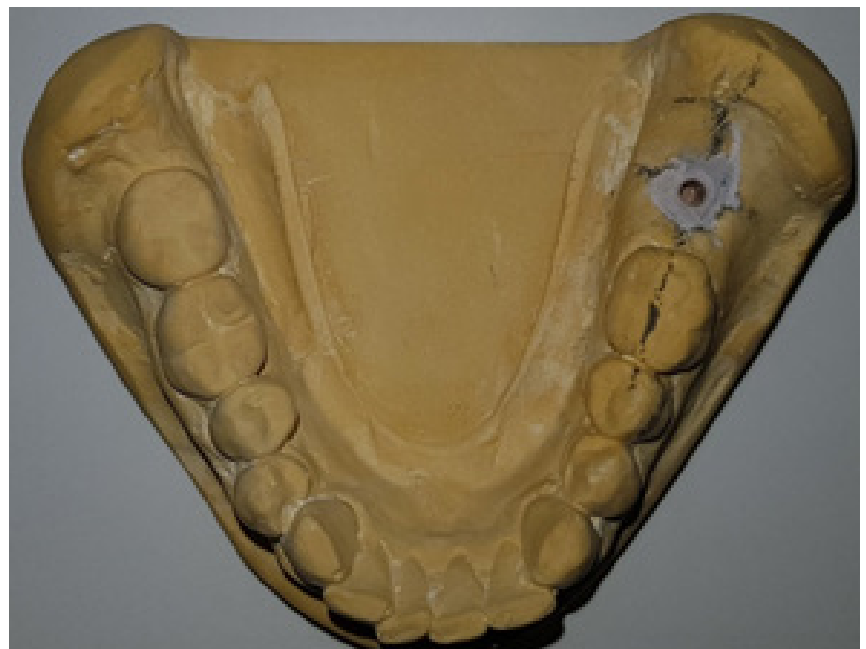

Figure. 2a. Centrepoint marked on the model

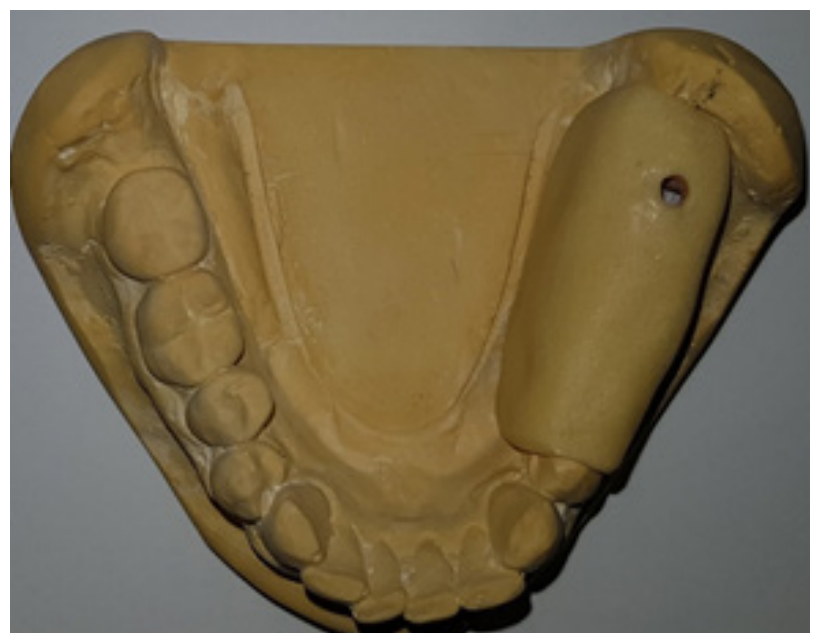

Figure 2b. Surgical guide on model
In this instance, the guide is designed to direct the pilot drill only into the correct spot so it is an "access guide". It gains its stability from teeth only making it an "access tooth supported guide."

Figure ' 3 ' shows a missing 12 tooth that will be implanted using a CAD/CAM designed SLA model made from composite resin. In this case, the guide is designed to guide the whole drilling and implant insertion process through the metal sleeve in the guide. This would be described as a: "precision tooth supported guide"

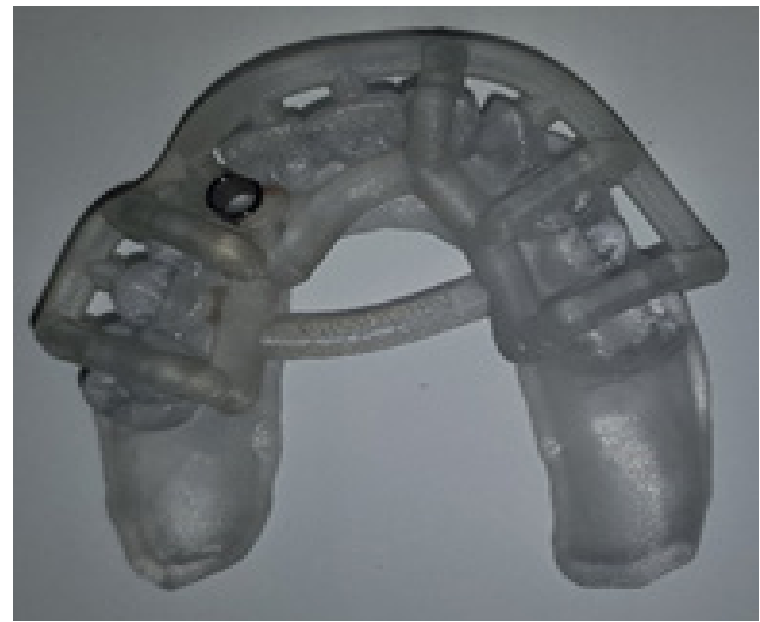

Figure. 3: Precision, tooth supported guide on model

Figure '4' shows another CAD/CAM guide manufactured from clear acrylic with metal sleeves for an edentulous maxilla. In this case, the guide gains retention from the tissues only.

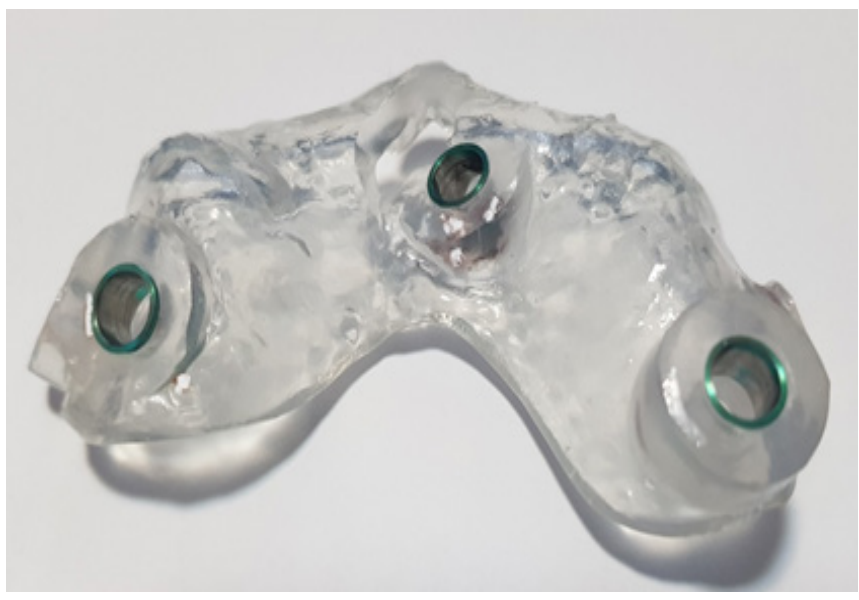

Figure 4: Precision tissue supported guide

\section{Discussion}

Current advancements in surgical techniques and enhanced placement technologies have contributed to the widespread utilization of dental implants as a viable mode of therapy. Following such extensive and, at times, complex den- 
tal implant treatments, the benchmark of patients' expectations from both the functional and aesthetic perspective have become extremely high.

Optimal implant placement is critical to the aesthetic and functional success of implant-supported restorations [12]. Not only is the accurate placement of the implant highly crucial in achieving a pleasing esthetic result, but, also, the correct alignment for withstanding occlusal forces is necessary for long term success [13].

It is clearly documented that the use of surgical guides for the placement of dental implants is more accurately positioned than those placed without a guide $[14,15]$. When planning for a surgical guide, the proper angulation and occlusal relationships are more readily assessable using dental casts where the lingual aspect is not obscured. In addition, a surgical guide also allows for less stressful surgery for the operator, knowing that the critical placement factors had already been accounted for in the fabrication of the guide [16].

Stumpel [10], argued that the use of surgical guides is beneficial from an operative perspective. All the decisions regarding implant positioning have been planned prior to surgery and hence, the surgery is just a matter of executing this plan. This is true if the guide is more restrictive where the implant placement procedure may only last for a few minutes and would allow flapless types of surgery. This would reflect positively on the patient with less postoperative comfort and faster healing.

The published articles on different designs of surgical guides are numerous. Most of those articles are case reports that explain the rationale and technique of constructing that particular guide. There is a full spectrum of techniques ranging from a simple suck-down template to technology-assisted designs to the stereolithographic computer manufactured models. This plethora of published information has led the standard practitioner to request a surgical guide and receive what the restorative dentist, in collaboration with his laboratory technician, was familiar with. This will not be necessarily the ideal guide that was expected due to the lack of a developed communication language secondary to the absence of an accepted universal classification of different surgical guides.

The available literature approaches the classification form three different perspectives. The first is the support of the guide perspective: bony versus gingival. Second, is the removable denture perspective: tooth supported versus tissue supported versus tooth and tissue supported which is, in a way, similar to the previous approach. Finally, the conceptual approach: non-limiting versus partially limiting versus completely limiting guide $[10,11,17]$. In a recent review, D'Souza and Aras [18] described the different types of surgical guides and followed the conceptual classification by Stumpel [10]. They further subdivided the completely limiting surgical guide into a cast based and a CADCAM based surgical guide.

None of those classifications is comprehensive or clear enough to serve as means of communication between the oral surgeon/periodontist, restorative dentist/prosthodontist and the laboratory technician making the guide. Furthermore, there are no easy means for the implanting practitioner to convey his/her desires about the nature of the guide to the restorative dentist or dental lab.

The characteristics of an ideal surgical guide as pointed out by Annibali and La Monaca [3] is that it should be stable, rigid, of limited size, easy to insert, transparent, modifiable and able to be sterilized. Even then, many of the published surgical guides do not fulfill those requirements.

The guide needs to be stable and in dentate patients, most of that can be obtained from the surrounding dentition. If the patient is edentulous then extensions may be necessary. This is not always practical if a sizeable surgical flap is raised and hence further means of stability is required. The other solution for this is to gain extra stability from the surrounding bone (not used for the implants) by using pins or screws [9]. Based on that, one parameter of the classifications need to incorporate the nature of the support of the guide which would be: tooth supported only, tooth and tissue supported, tissue supported only and tissue supported with accessory fixation.

To ensure further rigidity the material used for the construction of the splint is very important. Stumpel [10] summarized the three used materials: acrylic, composite resin, and VPS. Because of the malleability of VPS, only the most rigid of VPS materials should be used. The nature of the material used does not need to be part of the classification but it is important for all involved personnel to be aware of what is available and that could be communicated in the laboratory request card.

The conceptual classification of the guides is a very useful one and sheds a lot of light on the design of the guide but is one that may need some re-wording to serve a more communicable means. This classification really only makes good sense to the practitioner placing the dental implants but will certainly not be one that can be communicated to the dental technician on a lab card.

A non-limiting guide is one that will allow the implanting practitioner to simultaneously achieve the decision making and implant placement during the time of surgery. This concept was covered in the proposed classification as the: "free guide". This guide, ideally, needs to conform to the criteria described by Balshi and Garver ${ }^{11}$ namely, maintaining the 
labial surfaces of the maxillary teeth on the stent to allow the implant to be placed within the general cut-out area behind that surface. Regarding the mandible, the dental implant area would be located lingual to the incisal edges of the anterior denture teeth and within the confines of the occlusal surface of the posterior teeth.

The partially limiting guide will have a mechanism for the pilot drill to identify the dental implant site and the rest of the angulation will be controlled by the operator. These guides usually have a central hole that will guide the pilot drill but not really control it. This concept was covered in the proposed classification under the "access guide".

The completely limiting guide is the one that incorporates the metal guide sleeves that direct the implant exactly to where the preoperative planning has been made. Those have been termed in the proposed classification as "precision guide". The stereolithographic guides that are done using the CAD/CAM technology fall into this category but could, also, be manually constructed. During the surgical phase, the difference in the diameter of surgical drills can be accommodated by supplying multiple duplicate guides with sleeves of graded diameters or with adapter cylinders that fit inside the guide's sleeve or by using special drills with stoppers such as the MGuides provided by MIS implants.

It is, also, very important that the classification incorporates the support of the guide for two reasons. First, the stability of the guide is the most important technical factor for its successful use. Second, the operator may desire certain areas in the mouth be visible for other considerations such as flap design, bone grafting procedures, and drill angulations.

The classification in this study is, hence, one that consists of a $3 \times 4$ model. There are three surgical guide designs: free, access and precision and 4 support options: tooth, tissue, both, tissue with added fixation.

One last dimension from the surgeon's perspective required a further modification of the proposed classification. If a surgical guide is constructed manually without the computer-assisted technology, it may conceal the bony interface and without CAD/CAM technology this may become a major disadvantage. One of the published techniques to overcome this problem was the segmented surgical guide (fig. 5) described by Ma and Brudvik [19]. This guide consists of lingual and labial sections that are assembled and attached together as a single unit via tiny embedded, separable magnets. The lingual section, which can remain in place during surgery has the markings that describe the location and orientation of each implant. If any surgical bone modification is needed it can be done by removing the labial component. Once removed, the surgical site becomes readily apparent and facilitates access for surgical instruments.
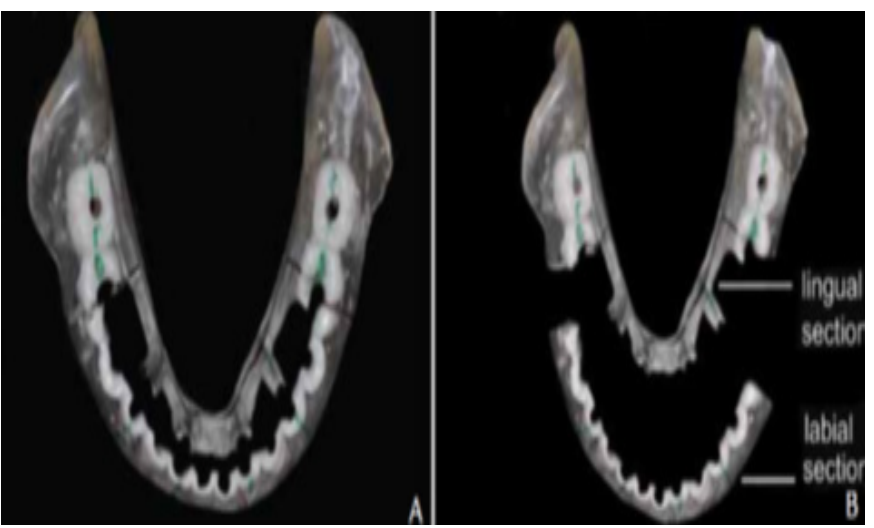

Figure 5: The segmented surgical guide (Ma and Brudvik, 2011).

A similar concept could be to have guide sleeves within the guide that are able to be removed and slotted back in during surgery or be split in the middle along their vertical plane as in the guide described by Wat et al [20], to increase surgical visibility. In order to incorporate those peculiar modifications the access and precision, surgical guides were further subdivided into ones "with fixed guidance or removable guidance" in the proposed classification. With this new classification in mind, lab card request forms for the upper (Figure. 6) and lower (Figure. 7) have been proposed.

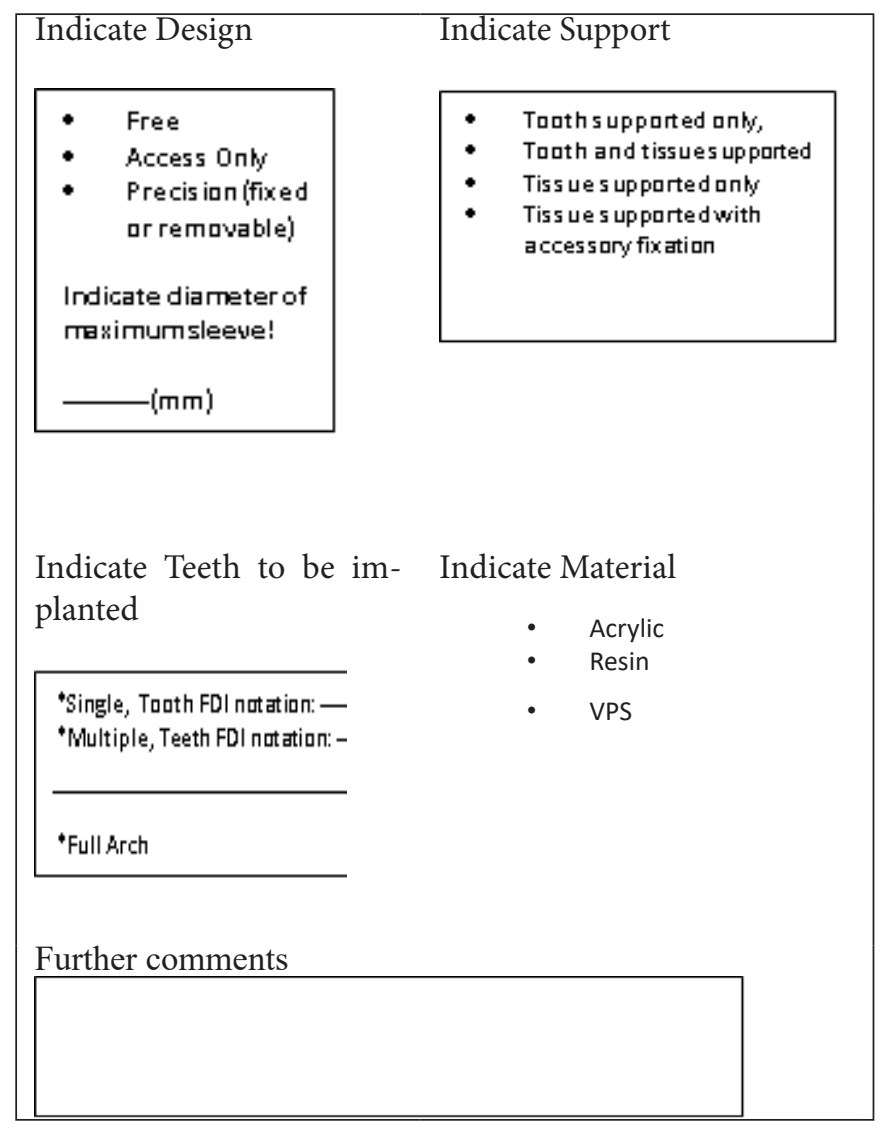

Figure 6: LAB CARD, Request for implant surgical guide(upper jaw) 


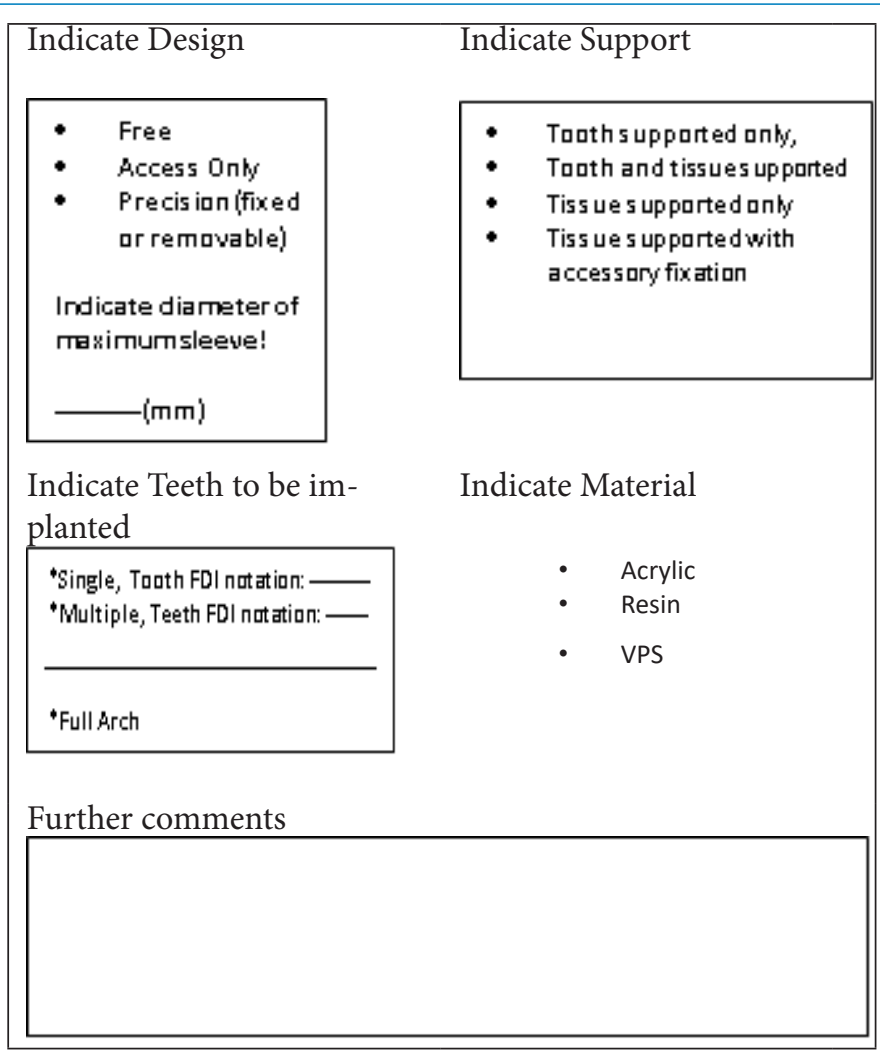

Figure 7: LAB CARD, Request for implant surgical guide(lower jaw)

\section{Conclusion}

It can be concluded that the surgical guides will continue to be a valuable adjunct to achieving precision in today's prosthetic driven implantology. The guides serve diagnostic and surgical purposes and depending on the complexity of the case may be manually constructed guides or stereolithographic ones. The conventional guides may be simply constructed or be made using advanced radiographic +/- computer technology i.e three-dimensional technology. The process of obtaining a surgical guide has no particular guidelines and is operator dependent. Given the number of professionals involved in treatment planning and execution, a great need for a specific language is warranted to communicate the laboratory needs. A classification of the surgical guides and instruction laboratory card, as suggested in this study, would hopefully serve that purpose.

\section{References}

1. Koyanagi K (2002) Development and clinical application of a surgical guide for optimal implant placement. J Prosthet Dent 88:548-552.

2. Drago C \& Peterson T (2010) Implant Laboratory Procedures: A Step-by-Step Guide Wiley-Blackwell, Singapore 33-40.

3. Annibali S, La Monaca G (2009)The role of the template in prosthetically guided implantology. J Prosthodont 18:177-183.

4. $\quad$ Adrian ED, Ivanhoe JR \& Krantz WA (1992) Trajectory Surgical Guide Stent for Implant Placement J Prosthet Dent 67:687-691.

5. Garber DA (1995) Restoration Driven Implant placement with restoration generated site development Compend Cont Edu Dent 16:796-804.

6. Garber DA (1996) The esthetic dental implant: letting restoration be the guide J Oral Implantol 22:45-50.

7. Monson ML (1994) Diagnostic and Surgical Guides for Placement of Dental Implants. J Oral Maxillofac Surg 52:642-645.

8. Ozan O, Orhan K \&Turkyilmaz, I (2011) Correlation Between Bone Density and Angular Deviation of Implants Placed Using CT-Generated Surgical Guides. J Craniofac Surg 22:1755-1761.

9. Simon H (2002) Use of transitional implants to support a surgical guide: enhancing the accuracy of implant placement. J Prosthet Dent 87:229-232.

10. Stumpel LJ (2008) Cast-based guided implant placement: A novel technique J Prosthet Dent 100:61-69.

11. Balshi TJ \& Garver DG (1987) Surgical Guide stents for Placement of Implants J Oral Maxillofac Surg 45:463-466.

12. Atsu SS (2006) A surgical guide for dental implant placement in edentulous posterior regions. J Prosthet Dent, 96:129-133.

13. Kopp KC, Koslow AH \& Abdo OS (2003) Predictable implant placement with a diagnostic/surgical template and advanced radiographic imaging. J Prosthet Dent 89:611-615.

14. Engelman MJ, Sorensen JA \& Moy P (1998) Optimum placement of osseointegrated implants. J Prosthet Dent 59:467473. 
15. Higginbottom FL \& Wilson TG (1996) Three-dimensional templates for placement of root-form dental implants: a technical note. Int J Oral Maxillofac Implant 11:787-793.

16. Sarment DP, Sukovic P \& Clinthorne N (2003) Accuracy of implant placement with a stereolithographic surgical guide. Int J Oral Maxillofac Implant 18:571-577.

17. Shafie HR (2007) Clinical and Laboratory Manual of Implant Overdentures Blackwell Munksgaard 26-27.

18. D'Souza KM, Aras MA (2012) Types of Implant Surgical Guides in Dentistry: A Review. J Oral Implantol 38:643652.

19. Ma J \& Brudvik JS (2011) A segmented implant surgical guide connected with magnets. J Prosthet Dent 106:272276.

20. Wat PY, Chow TW, Luk HW \& Comfort, MB (2002) Precision surgical template for implant placement: a new systematic approach. Clin Implant Dent Rel Res 4:88-92.

Submit your manuscript to a JScholar journal and benefit from:

ฯ Convenient online submission

ฯ Rigorous peer review

ब Immediate publication on acceptance

q Open access: articles freely available online

I High visibility within the field

ब Better discount for your subsequent articles

Submit your manuscript at http://www.jscholaronline.org/submit-manuscript.php 